\title{
An Improved Sparse Channel Estimation for OFDM Systems Based on Sparse Reconstruction by Separable Approximation
}

\author{
Xiaolin Shi ${ }^{1,2}$ and Yixin Yang ${ }^{2}$ \\ ${ }^{1} X i$ 'an University of Posts and Telecommunications, Xi'an 710121, P. R. China \\ ${ }^{2}$ Northwestern Polytechnical University, Xi'an 710072, P. R. China \\ linda20016@163.com
}

\begin{abstract}
In high-rate data or multi-antenna orthogonal frequency division multiplex (OFDM) scenarios, many encountered channels trend to have the structure of sparse multi-paths. The communication systems over multipath channels usually require that the channel response be known at the receiver and thus channel estimation is obligatory. In this paper, we focus on exploring a channel estimator by exploiting the sparse features of multipath fading channels for OFDM communication systems. At the same time, a novel scheme based on the sparse reconstruction by separable approximation (SpaRSA) for the sparse channel estimation is presented to improve the poor performance of the typical least square $(L S)$ and $\ell_{2}$-norm channel estimations. Compared with the greedy pursuit algorithm, such as matching pursuit (MP) and orthogonal matching pursuit (OMP), the algorithm is able to reach the global optimal solution. By applying a threshold to determine the non-zero taps and introducing the noise effect into the regularization parameter in the SpaRSA algorithm, this proposed scheme leads to a superior estimation performance. The complexity of the proposed algorithm is lower than the SpaRSA algorithm because only a few channel taps are needed to recover with high accuracy considering the nature of sparse channels. In the simulation section, the proposed method is compared and contrasted with the existing estimation methods. And the simulation results show the validity of the proposed approach.
\end{abstract}

Keywords: Sparse channel estimation, sparse reconstruction by separable approximation, orthogonal frequency division multiplex

\section{Introduction}

In OFDM communication systems, signals transmitted via other reflectors or diffraction are severely attenuated, and arrive at the receiver as a superposition of multiple copies of the transmitted signal, called multipath signal components. This multipath signal propagation leads to fading in the received signal strength that severely affects the rate and reliability of wireless communication systems [1]. However, if the knowledge of channel state information (CSI) is available at the receiver, it enables the exploitation of delay, Doppler, and/or spatial diversity to combat fading while further gains in rate and reliability are possible as well [1][2]. In practice, CSI is seldom available to communication systems a priori and the channel impulse response (CIR) needs to be (periodically) estimated at the receiver in order to gain the reliable copy of the transmitted signals.

Many wireless channels encountered in practice tend to exhibit a sparse multipath structure at high signal space dimension [3]-[5]. Such sparse multipath communication channels include cellular communication environments, underwater acoustic channels, aeronautical communication channels, high-frequency radio communication channels and terrestrial high-definition television broadcasting channels. For this type of channels, the majority of channel taps are zero-value taps and the number of the most significant taps, 
which capture dominant channel power, is far less than the maximum number of excess delay.

Because of the variation of the number and position of non-zero taps, it becomes a challenge to perform the channel estimation with robust and prominent performance. The sophisticated least-squared (LS) algorithm [6] does not need the real-time computation of matrix inversion and assumes minimal a priori knowledge about the unknown channel because it estimates all the channel coefficients directly. But, in sparse channels, the estimation accuracy of LS is worse than that of the algorithms tailored for sparse channel estimation [2][7-12]. MP algorithm [7] is a typical representative of the sparse channel estimation algorithms. It sequentially takes a strongly correlated column of dictionary matrix with the residual signal and estimates the corresponding channel taps. By prespecifying the stopping rule reasonably, the algorithm can remove the noise in some of zero taps. Therefore it achieves a substantial reduction in estimation error compared with LS algorithm in sparse channels. By avoiding re-selecting the basis vectors, OMP algorithm has been shown to achieve much better performance than MP algorithm [11]. In [12], an iterative detector (IDE) scheme with threshold attempts to estimate the locations of non-zero channel taps by transforming the tap detection problem into an equivalent onoff keying detection problem. A modified iterative detector/estimator (MIDE) algorithm [13] has improved performance by applying an iteration-varying threshold instead of multiple thresholds in parallel for each iteration in IDE algorithm. IDE and MIDE offer different tradeoffs between performance and complexity, in the case of appropriate stopping rules of zero-tap search. However, these algorithms [7-12] are sensitive to the choice of the stopping rules. And in some multipath scenarios, these algorithms may result in significant performance degradation, which are even worse than LS algorithm [6].

In addition, a threshold-based approach [14] for sparse channel estimation in orthogonal frequency division multiplexing (OFDM) systems (named as TMSE) is derived by minimizing mean squared error (MSE) per CIR coefficient and these thresholds are then applied on the CIR LS estimate to detect its structure. Unfortunately, MSE is very sensitive to tap missing and false detection that is likely to degrade the detection performance for channel taps. In Bayesian statistics, a maximum a posteriori (MAP) algorithm [15] can obtain an accurate estimate on the basis of a priori knowledge of channel probability distribution. However, the priori knowledge is usually difficult to be obtained in practice.

The SpaRSA algorithm [16] based on the basis pursuit denoising (BPDN) in [17] is used to find sparse approximate solutions to large under-determined linear equations. It is an algorithmic scheme that is one of the fastest algorithms for solving a $\ell_{2} \ell_{1}$ optimization subproblem and can reach the optimal solution [16][18]. The regularization parameter in SpaRSA algorithm, which balances the sparsity of signals against the residual error, is very important for the whole algorithm. However, it was set to be a fixed number in the original algorithm [16] and hence cannot reflect the noise effect. As noise level varies, the estimation performance would suffer degradations.

Considering the nature of sparse channels, the estimation efforts can be focused on a small number of non-zero taps after the position of non-zero taps are detected, thus leading to a more accurate estimate of the channel coefficients. To achieve this goal, a threshold derived from the raw LS estimate is presented to determine the position of nonzero taps. Then, the estimates of channel taps are further refined by the improved SpaRSA algorithm, which is obtained by introducing the noise effect into the regularization parameter. With the use of this threshold and the proposed regularization parameter, the whole algorithm enhances the estimation efficiency and hence improves the estimation accuracy.

The rest of this paper is organized as follows. We present the OFDM system model in Section II. In Section III, we address the sparse estimation formulations. Section IV 
proposes the novel channel estimation scheme and presents detailed steps of the proposed algorithm. In Section V, the performance of the proposed method is verified through simulations. And we conclude the paper in Section VI.

Notations used throughout this paper are based on the convention. Vectors are represented as boldface lowercase letters and matrices as boldface uppercase letters. $(\cdot)^{T}$, $(\cdot)^{H},(\cdot)^{-1},(\cdot)^{+},\|\cdot\|^{0},\|\cdot\|^{1}$ and $\|\cdot\|^{2}$ denote the transpose, the Hermitian transpose, the inverse, the pseudo-inverse, $\ell_{0}$-norm, $\ell_{1}$-norm, $\ell_{2}$-norm, respectively.

\section{OFDM System Model}

Consider a pilot-assisted OFDM system with $N$ subcarriers in each symbol, among which $N_{d}$ data symbols are used for delivering messages and $N_{p}$ pilot symbols indicated by $p_{1}, p_{2}, \ldots, p_{N p}$ are used for pilot-assisted channel estimation. The subcarriers are modulated by symbols mapped from QAM or PSK constellation. After OFDM modulation, $L_{c p}$ samples of the cyclic prefix $(\mathrm{CP})$ are added at the beginning of the $N$ samples of inverse discrete Fourier transform (IDFT) to form one OFDM symbol of $\mathrm{N+}$ $L_{c p}$ samples, which can be written as

$$
x_{n}=d_{n}+p_{n}, \quad-L_{c p} \leq n \leq N-1 .
$$

Here $d_{n}$ and $p_{n}$ represent the data and pilot symbols, respectively.

Since a variety of channels are prone to multipath propagation due to refraction, reflection, and scattering, the channel impulse response $h(k)$ has the form

$$
h(k)=\sum_{l=0}^{L-1} a_{l} \delta\left(k-\tau_{l}\right),
$$

where $\delta(\cdot)$ is the delta function. $a_{l}$ and $\tau_{l}$ is the amplitude and time-delay of the $l$ th path, respectively. In the above model, path delays are sample spaced and $L$ denotes the delay spread of a channel. The OFDM symbols are then transmitted over a multipath fading channel. Assume that the $\mathrm{CP}$, with the length $L_{c p}$ samples, is longer than the maximum path delay of the channel $L$ in order to guarantee the orthogonality of subcarriers of the OFDM system, i.e., $L_{\mathrm{cp}}>L$, and that the synchronization is perfect at the receiver.

Then, at the receiver the remaining samples, after the $\mathrm{CP}$ is removed, are demodulated by the $\mathrm{N}$-point discrete Fourier transform (DFT) operation. So, the received sample vector is expressed as

$$
\mathbf{y}=\mathbf{X F H}+\mathbf{n}_{f},
$$

where $\mathbf{y}=\left[y_{0}, y_{1}, \ldots, y_{N-1}\right]^{T}$ is the received symbols. $\mathbf{X}$ is the $N \times N$ diagonal matrix of transmitted data comprising data matrix $\mathbf{D}$ and pilot matrix $\mathbf{P}$, i.e., $\mathbf{X}=\mathbf{D}+\mathbf{P}=$ $\operatorname{diag}\left[x_{0}, x_{1}, \ldots, x_{N-1}\right]^{T} . \mathbf{F}$ is $N \times N$ DFT matrix with entires $F_{l, m}=1 / \sqrt{N} e^{\frac{-j 2 \pi(l-1)(m-1)}{N}}$, where $l, m=1,2, \ldots, N$ and the channel vector $\mathbf{H}=[h(0), \ldots, h(L-1), 0, \ldots, 0]^{T}$. The zero-mean complex Gaussian noise vector $\mathbf{n}_{f}$ has its covariance matrix $\sigma_{f}^{2} \mathbf{I}_{N}$, i.e., $\mathbf{n}_{f} \sim N\left(0, \sigma_{f}^{2} \mathbf{I}_{N}\right)$.

Generally, the received samples of pilot symbols are used for the channel estimation, which can be expressed as

$$
\mathbf{r}=\mathbf{P F} \mathbf{F}_{p} \mathbf{h}+\mathbf{n}=\mathbf{A} \mathbf{h}+\mathbf{n},
$$

i.e. 


$$
\left[\begin{array}{c}
r(0) \\
r(1) \\
\vdots \\
r\left(N_{p}-1\right)
\end{array}\right]=\left[\begin{array}{cccc}
p_{1} & 0 & 0 & 0 \\
0 & p_{2} & 0 & 0 \\
0 & 0 & \ddots & 0 \\
0 & 0 & 0 & p_{N p}
\end{array}\right] \cdot \mathbf{F}_{p} \cdot\left[\begin{array}{c}
h(0) \\
h(1) \\
\vdots \\
h(L-1)
\end{array}\right]+\left[\begin{array}{c}
n(0) \\
n(1) \\
\vdots \\
n\left(N_{p}-1\right)
\end{array}\right]
$$

where $\mathbf{A}=\mathbf{P F} \mathbf{F}_{p}$ is usually known as measurement matrix. $\mathbf{P}$ is the $N_{p} \times N_{p}$ diagonal matrix of the pilots. The channel vector $\mathbf{h}=[h(0), h(1), \ldots, h(L-1)]^{T}$ and $\mathbf{n}$ is the additive white Gaussian noise vector. In practice, since the sampling period is usually much smaller than the channel delay spread, most components of $\mathbf{h}$ are either zero or nearly zero, meaning that $\mathbf{h}$ is sparse vector. $\mathbf{F}_{p}$ is a $N_{p} \times L$ matrix formed by the first $L$ columns of the DFT matrix $\mathbf{F}$ in (3) and the $N_{p}$ rows of the selected matrix associated with the pilot subcarriers, which is written as

$$
\mathbf{F}_{p}=\frac{1}{\sqrt{N}}\left[\begin{array}{cccc}
1 & \omega^{p_{1}} & \cdots & \omega^{p_{1} \cdot(L-1)} \\
1 & \omega^{p_{2}} & \cdots & \omega^{p_{2} \cdot(L-1)} \\
\vdots & \vdots & \ddots & \vdots \\
1 & \omega^{p_{N p}} & \cdots & \omega^{p_{N p} \cdot(L-1)}
\end{array}\right],
$$

where $\omega=e^{-j 2 \pi / N}$.

\section{Sparse Estimation Formulations}

In the literature, the sum of the non-zero taps is often used to measure the sparseness of the channel, i.e., the $\ell_{0}$-norm of $\mathbf{h}$ :

$$
\|\mathbf{h}\|_{0}=\sum_{i=0}^{L} 1\left(h_{i} \neq 0\right)
$$

where $1\left(h_{i} \neq 0\right)$ denotes that if $h_{i} \neq 0$, the value of the function $1(\cdot)$ equals to 1 ; otherwise it equals to 0 . From (13), the $\ell_{0}$-norm of $\mathbf{h}$ is equal to the number of non-zero taps, $D$. So, the sparse channel estimation comes down to solving the following optimization problem:

$$
\min _{h}\|\mathbf{h}\|_{0} \quad \text { s.t. }\|\mathbf{r}-\mathbf{A h}\|_{2}^{2} \leq \varepsilon,
$$

where $\varepsilon$ is a non nonnegative real parameter. The above equation is a non-convex optimization problem and it is hard to obtain its solutions. To solve the problem, the following formulation

$$
\min _{h}\|\mathbf{r}-\mathbf{A h}\|_{2}^{2} \quad \text { s.t. }\|\mathbf{h}\|_{1} \leq T,
$$

is frequently used. Under certain conditions, $\ell_{1}$-norm is equal to $\ell_{0}$-norm [19]. Therefore, we have

$$
\min _{h}\|\mathbf{h}\|_{1} \quad \text { s.t. }\|\mathbf{r}-\mathbf{A h}\|_{2}^{2} \leq \varepsilon,
$$

where $T$ are nonnegative real parameters. This formula, however, is not taken noises into account. As an improvement, the optimization problem with the consideration of noises can be expressed as: 


$$
\min _{h} \frac{1}{2}\|\mathbf{r}-\mathbf{A h}\|_{2}^{2}+\kappa\|\mathbf{h}\|_{1} .
$$

where $\kappa$ is a nonnegative real parameter and is referred to as the regularization parameter that balances the permissible errors and the signal sparsity. The above equation is the now famous $\ell_{2}-\ell_{1}$ problem. To solve this problem, we will propose the following algorithm.

\section{Proposed Sparse Channel Estimation Method}

\subsection{Sparse Channel Estimation}

The LS estimator for channel impulse response $\mathbf{h}$ is given by

$$
\hat{\mathbf{h}}_{L S}=\mathbf{A}^{+} \mathbf{r}=\left[\mathbf{A}^{H} \mathbf{A}\right]^{-1} \mathbf{A}^{H} \mathbf{r},
$$

where $\mathbf{A}^{+}=\left[\mathbf{A}^{H} \mathbf{A}\right]^{-1} \mathbf{A}^{H}$ is the pseudo-inverse of $\mathbf{A}$. Substituting (4) into (12), the above equation is expressed as

$$
\hat{\mathbf{h}}_{L S}=\mathbf{h}+\left[\mathbf{A}^{H} \mathbf{A}\right]^{-1} \mathbf{A}^{H} \mathbf{n} .
$$

We consider the case that the pilots are uniformly distributed and $\mathbf{A}^{H} \mathbf{A}=N_{p} \mathbf{I}$, the equation of (13) can be rewritten as:

$$
\hat{\mathbf{h}}_{L S}=\mathbf{h}+\frac{1}{N_{p}} \mathbf{A}^{H} \mathbf{n}=\mathbf{h}+\mathbf{v} .
$$

where $\mathbf{v}=\left(1 / N_{p}\right) \mathbf{A}^{H} \mathbf{n}$ and it is still a zero-mean complex Gaussian vector with its covariance matrix $\mathbf{C}_{m}$ expressed as:

$$
\mathbf{C}_{m}=E\left(\mathbf{v v}^{H}\right)=\sigma_{f}^{2} \mathbf{I} / N_{p}=\sigma_{v}^{2} \mathbf{I},
$$

where $\sigma_{v}^{2}$ is the standard deviation of the noise vector $\mathbf{v}$. Based on the observation that the channel length is shorter than the CP duration, the first $L_{c p}$ components of the raw LS estimate $\hat{\mathbf{h}}_{L S}$ can be viewed as the initial estimate of the sparse channel vector $\hat{\mathbf{h}}$ :

$$
\hat{\mathbf{h}}_{L S}=\mathbf{h}+\mathbf{v}=\left(\begin{array}{c}
\mathbf{h}+\mathbf{v}^{\prime} \\
\mathbf{0}+\mathbf{v}^{\prime \prime}
\end{array}\right)=\left(\begin{array}{c}
\hat{\mathbf{h}} \\
\mathbf{0}+\mathbf{v}^{\prime \prime}
\end{array}\right) .
$$

where $\mathbf{v}=\mathbf{v}^{\prime}+\mathbf{v}^{\prime \prime}$.

Obviously seen from (14), the estimated results of CIRs are fully contaminated by noise. Therefore, we essentially have a binary hypothesis test for the channel tap:

$$
\left\{\begin{array}{l}
H_{0 i}: h_{i} \cdot 0+\left.v_{i}\right|_{b_{i}=0}=v_{i} \\
H_{1 i}: h_{i} \cdot 1+\left.v_{i}\right|_{b_{i}=1}=h_{i}+v_{i}
\end{array},\right.
$$

where $H_{0 i}$ indicates that $h_{i}$ is a zero tap in the $i$ th tap position whereas $H_{l i}$ indicates that $h_{i}$ is a non-zero tap in the $i$ th tap position. Note that the above hypothesis test is independent of the number of non-zero taps, which render the consequent detection of channel taps.

In order to build a detectable strategy for non-zero taps, we have the following expression as:

$$
\mathbf{r}=\mathbf{A d i a g}(\mathbf{b}) \mathbf{h}+\mathbf{n} \text {. }
$$


The non-zero values of the channel vector $\mathbf{h}$ are in the set of positions $\Theta=\left(z_{0}, z_{1}, \ldots, z_{D-1}\right)$. The total number of non-zero taps in the CIRs, $D$, is unknown $a$ priori and generally $D<L<L_{c p}$ for sparse channels. b is a sparse vector, which indicates the sparse structure of the channels and its entries are given by

$$
b_{i}=\left\{\begin{array}{lr}
1, \quad i \in \Theta \\
0, \text { otherwise } .
\end{array}\right.
$$

To detect the $D$ non-zero taps out of the initial estimate of the sparse channel vector $\hat{\mathbf{h}}$, the universal threshold $\Gamma$ proposed in [20] for compressed channel sensing is applied as

$$
\Gamma=\sqrt{4 \sigma_{v}^{2} \log L_{c p}} .
$$

From the above equation, we propose to estimate $\sigma_{v}^{2}$ from the noisy part of the raw LS estimate of CIR, which is written as:

$$
\hat{\sigma}_{v}^{2}=\frac{1}{N_{p}-L_{c p}} \sum_{k=L_{c p}+1}^{N_{p}}\left|\hat{\mathbf{h}}_{L S(k)}\right|^{2} .
$$

Hence, the threshold can be re-written as:

$$
\Gamma \square \sqrt{4 \hat{\sigma}_{v}^{2} \log L_{c p}} .
$$

Then, the processed initial estimate of the sparse channel vector $\hat{\mathbf{h}}$ can be determined as

$$
\hat{h}(l)=\left\{\begin{array}{cl}
\hat{h}(l), & |\hat{h}(l)|>\Gamma ; \text { non-zero taps } \\
0, & |\hat{h}(l)| \leq \Gamma ; \text { zero taps }
\end{array} \quad 0 \leq l \leq L_{c p}-1 .\right.
$$

Since the estimation accuracy of LS method is not satisfactory for sparse channels, we will make use of the SpaRSA algorithm, one of the fastest algorithms for solving the $\ell_{2}-\ell_{1}$ problem, to refine the selected non-zero taps in the processed initial estimate of $\hat{\mathbf{h}}$.

\subsection{Improved SpaRSA Algorithm}

The SpaRSA is an algorithmic framework that has comparable computational speed with the state-of-the-art algorithms used in solving the standard $\ell_{2}-\ell_{1}$ problems of (11), which is written as:

$$
\min _{h} \varphi(h)=f(h)+\kappa c(h),
$$

where

$$
\begin{array}{r}
f(h)=\frac{1}{2}\|\mathbf{r}-\mathbf{A h}\|_{2}^{2}, \\
c(h)=\|\mathbf{h}\|_{1} .
\end{array}
$$

In the SpaRSA method, a subproblem is set up and solved efficiently at each iteration. For the $\ell_{1}$ regularization problem, the subproblem has the following equivalent form

$$
\mathbf{h}^{t+1} \in \arg \min _{z} \frac{1}{2}\left\|\mathbf{z}-\mathbf{u}^{t}\right\|_{2}^{2}+\frac{\kappa}{\alpha_{t}} c(\mathbf{z}),
$$


where

$$
\mathbf{u}^{t}=\mathbf{h}^{t}-\frac{1}{\alpha_{t}} \mathbf{A}^{T}\left(\mathbf{A} \mathbf{h}^{t}-\mathbf{r}\right)
$$

When $c(\mathbf{z})=\|\mathbf{z}\|_{1}$, the unique minimizer is given by

$$
\arg \min _{z} \frac{\left(z-u_{i}^{t}\right)^{2}}{2}+\frac{\kappa|z|}{\alpha_{t}}=\operatorname{soft}\left(u_{i}^{t}, \frac{\kappa}{\alpha_{t}}\right),
$$

where $\operatorname{soft}(u, a) \equiv \operatorname{sign}(u) \max \{|u|-a, 0\}$ is the well-known soft-threshold function.

Choosing a proper value of the regularization parameter $\kappa$ is vital to SpaRSA algorithm. The selection of $\kappa$ will allows the $\ell_{2}-\ell_{1}$ formulation to recover the solution with high accuracy. As seen in [16], the unique solution to (29) is the zero vector if $\kappa \geq\left\|\mathbf{A}^{T} \mathbf{r}\right\|_{\infty}$, thus the regularization parameter must be selected within the scope of $(0$, $\left.\left\|\mathbf{A}^{T} \mathbf{r}\right\|_{\infty}\right)$.

In [16], $\kappa$ is set to $0.1\left\|\mathbf{A}^{T} \mathbf{r}\right\|_{\infty}$. However, this strategy cannot reflect the change of noise effect. So, we propose a parameter strategy as:

$$
\kappa=0.1 \times \sigma_{v}^{2} \times\left\|\mathbf{A}^{T} \mathbf{r}\right\|_{\infty},
$$

which can help the algorithm adapt with the noise change. With the estimate of $\sigma_{v}^{2}$ in (21), the above equation can be written as:

$$
\kappa \square 0.1 \times \hat{\sigma}_{v}^{2} \times\left\|\mathbf{A}^{T} \mathbf{r}\right\|_{\infty} .
$$

Based on the above expressions, the main steps of the proposed channel estimation method are summarized as the following steps:

Step 1: Initialize the channel estimation $\hat{\mathbf{h}}^{0}$ by the first $L_{c p}$ components of the raw LS estimate $\hat{\mathbf{h}}_{L S}$ in (12)

Step 2: Calculate $\hat{\sigma}_{v}^{2}$ by (21) and the threshold $\Gamma$ by (22).

Step 3: Update $\hat{\mathbf{h}}^{0}$ by (23).

Step 4: Use the improved SpaRSA algorithm to refine the non-zero taps in $\hat{\mathbf{h}}^{0}$ :

4-1. Choose $\eta$ and constants $\alpha_{\min }$ and $\alpha_{\max }$;

4-2. Choose $\alpha_{t} \in\left[\alpha_{\min }, \alpha_{\max }\right]$;

4-3. Choose $\kappa$ by (31);

4-4. Calculate $\hat{\mathbf{h}}^{t+1}$ by the subproblem (27);

4-5. Set $\alpha_{t+1}=\eta \alpha_{t}$ and if $\left\|\hat{\mathbf{h}}^{t+1}-\hat{\mathbf{h}}^{t}\right\|^{2}>\varepsilon$, increase $t$ and return to (4-2). Otherwise, the current estimate $\hat{\mathbf{h}}^{t+1}$ is the solution of CIR estimation.

Note that the parameters $\alpha_{\max }=1 / \alpha_{\min }=10^{30}$ and $\eta=2$ are set for our simulations. In this algorithm, the prior information of the number of non-zero channel taps is not required. This property will benefit the practical application of this algorithm as well. 


\section{Simulation Results and Analysis}
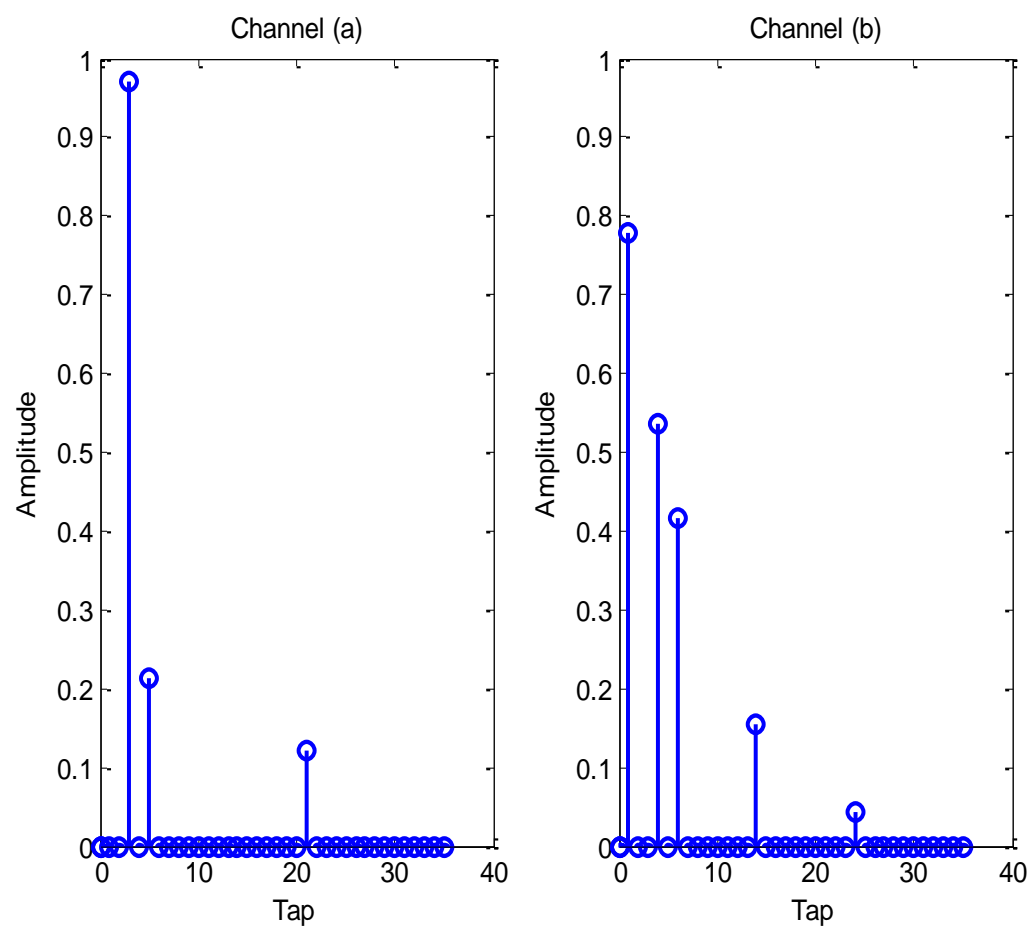

Figure 1. The Impulse Response of Channel (a) and Channel (b)

In this section, we do the simulation experiments that demonstrate the competitive performance of the proposed channel estimation approach. A 16QAM modulated OFDM system we consider in simulations has $N=512$ subcarriers and $N_{p}=128$ pilot subcarriers. $L_{c p}$ is set to 64 symbols. The length of channel impulse responses is $L=35$. The channel estimation algorithms are compared using the following channel models, which are illustrated in Figure 1: (a) 3-tap sparse channel in [21]; (b) 5-tap sparse Rayleigh fading channel with exponentially decaying power profile, $p(l)=e^{-0.125 l}$. This type of channel model (b) is widely used in many literatures. Both channels are assumed to be timeinvariant over the OFDM symbol duration. The channel power is normalized, that is, $\mathbf{h}^{H} \mathbf{h}=1$, and thus, the signal-to-noise ratio (SNR) is equal to $\mathbf{h}^{H} \mathbf{h} / \sigma_{v}^{2}=1$. In the simulations, the performance of different channel estimators is evaluated in terms of the MSE, defined as

$$
\operatorname{MSE}(\mathrm{dB})=10 \log _{10}\left(1 / N_{m} \sum_{n=1}^{N_{m}}\|\hat{\mathbf{h}}-\mathbf{h}\|^{2}\right)
$$

where $N_{m}$ is the total number of Monte Carlo iterations. 


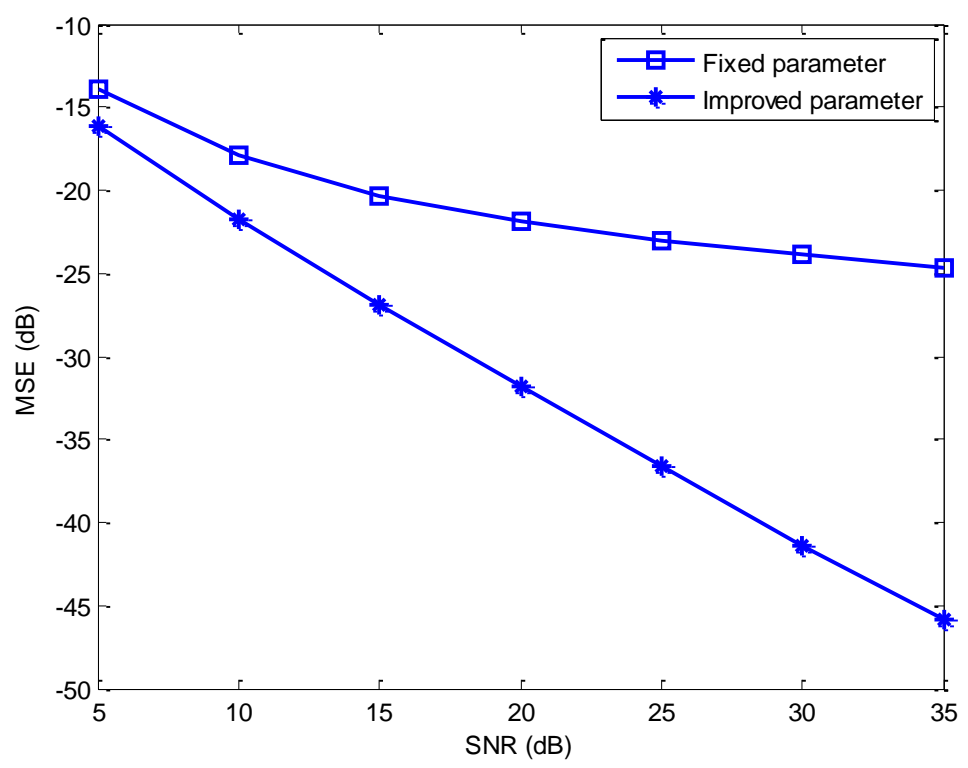

Figure 2. MSE Comparison of Channel (a) with Different Regularization Parameters

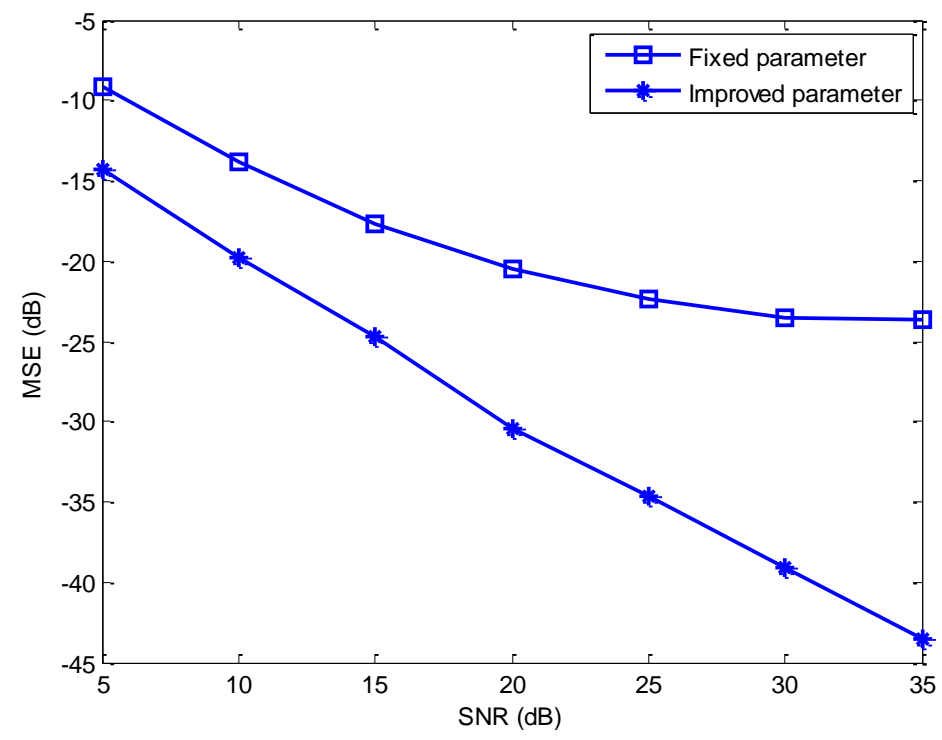

Figure 3. MSE Comparison of Channel (b) With Different Regularization Parameters

To access the effectiveness of the improved regularization scheme proposed in Section IV, we compare its MSE performance with the SpaRSA algorithm [16] using the fixed regularization parameter $\kappa=0.1\left\|\mathbf{A}^{T} \mathbf{r}\right\|_{\infty}$ under the two channel conditions in Figure 2 and Figure 3. The results in Figure 2 and Figure 3 show that when the regularization parameter is fixed the MSE trends to flat with the increase of SNRs. But the MSE decreases dramatically with the increase of SNRs when the regularization parameter is improved by introducing the noise effect. The reason is that when noise level decreases the regularization parameter should decrease in order to improve the accuracy of channel 
estimation. But the noise change effect did not reflected in the regularization parameter of the original SpaRSA algorithm.

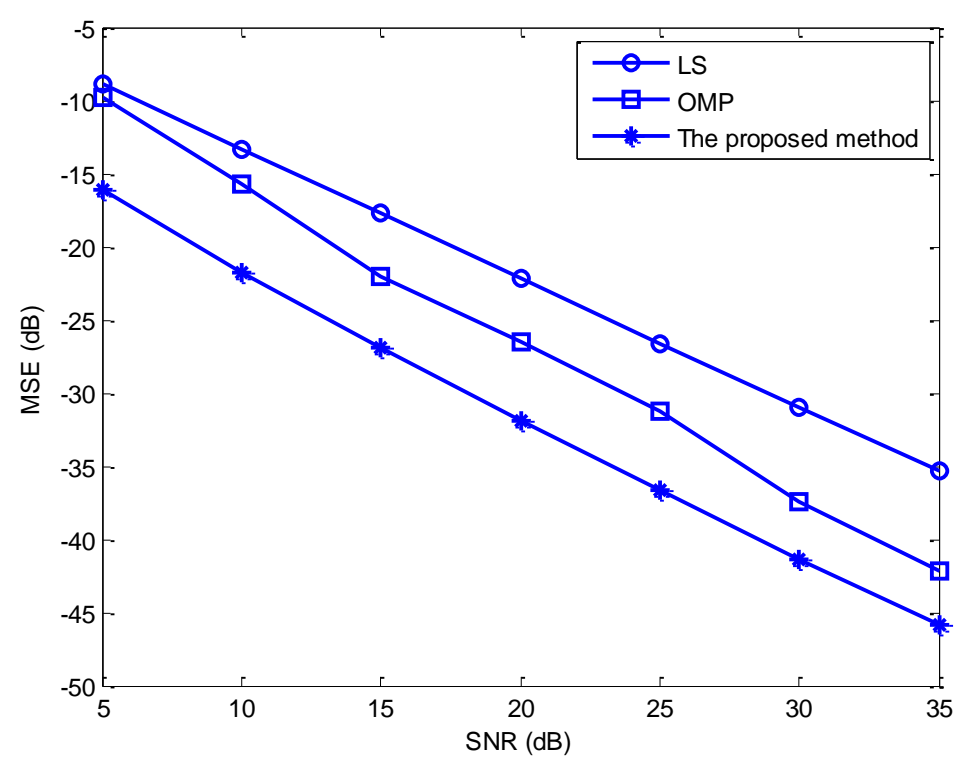

Figure 4. MSE Comparison of Channel (a) for Different Estimators

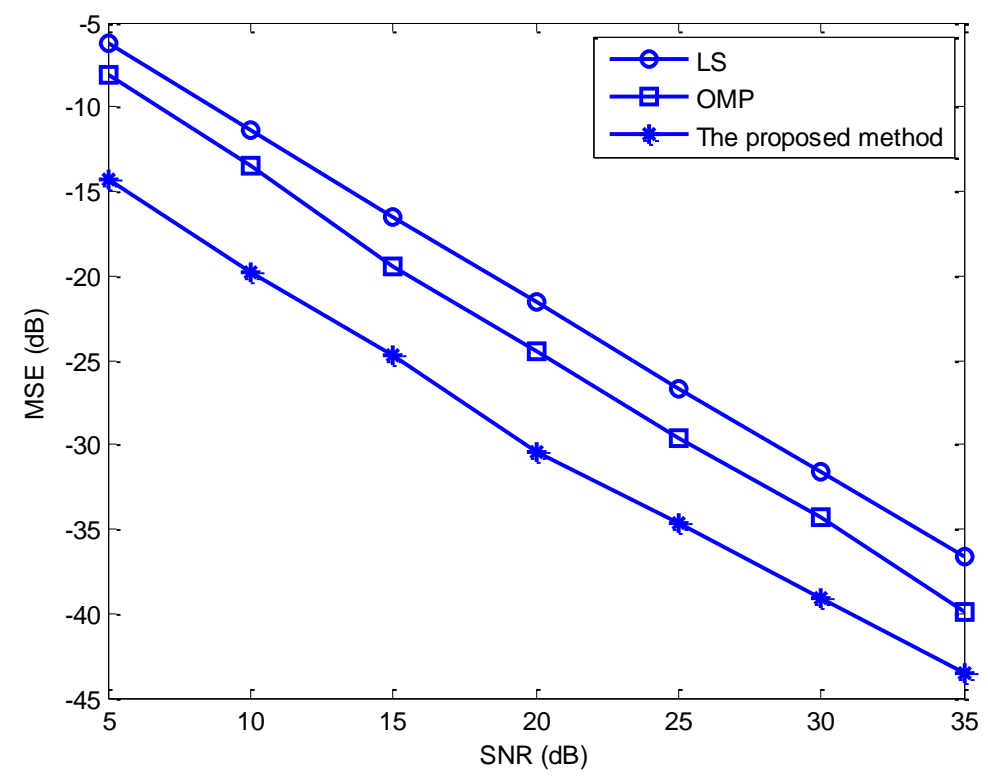

Figure 5. MSE Comparison of Channel (b) for Different Estimators

To test the performance of the proposed algorithm, the MSE of LS [6], OMP [11] and the proposed estimation algorithm are compared under two different channel conditions and averaged over 2000 Monte Carlo iterations for each SNR in Figure 4 and Figure5. The SNR variation ranges from 5 to $35 \mathrm{~dB}$. From Figure 4 and Figure 5, the proposed method has the best performance among all the estimators. Since it introduces the noise effect into the regularization parameter and applies the threshold $\Gamma$ to determine the nonzero taps, the accuracies of the proposed method are superior to those of other methods. 
The OMP algorithm results in significant performance degradation as the number of nonzero taps increases in that it is very difficult to choose a good stopping rule [13][21].

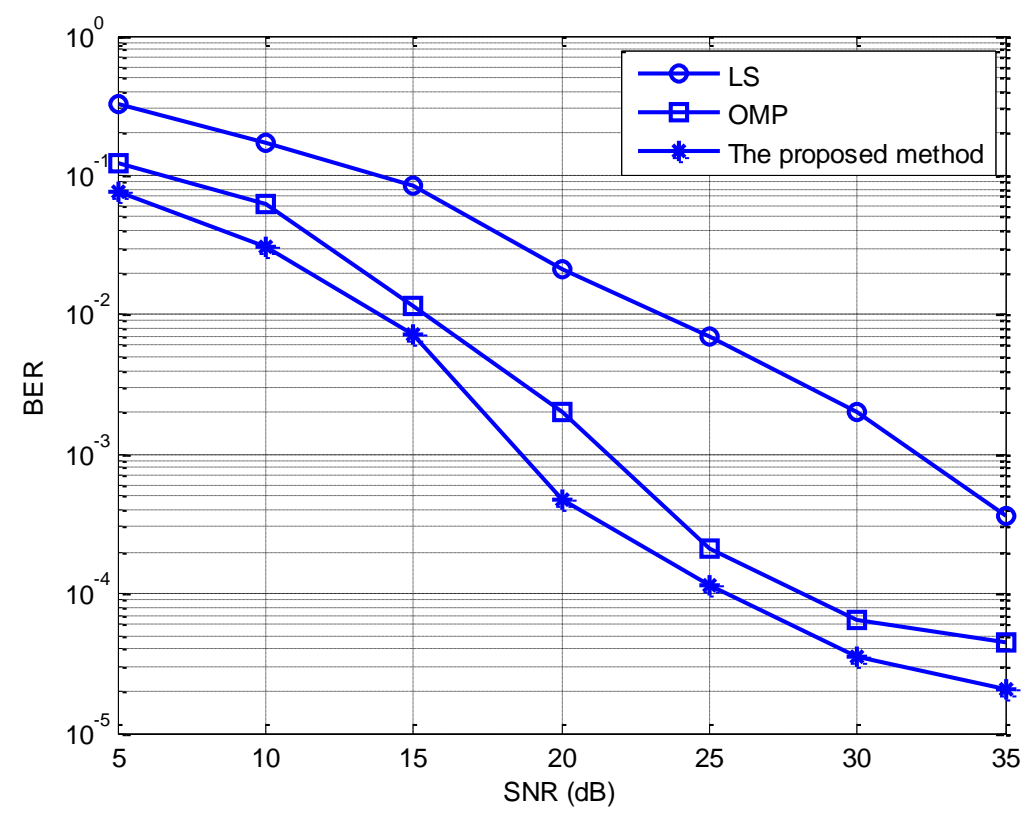

Figure 6. BER Comparison of Channel (a) for Different Estimators

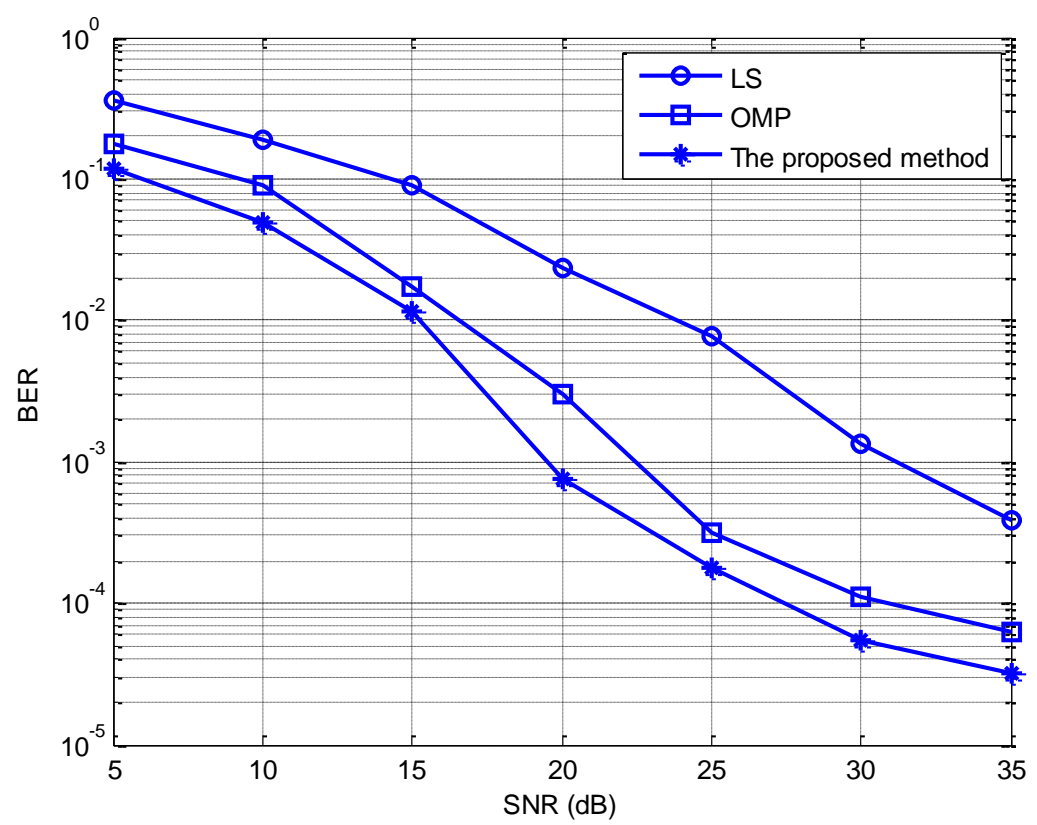

Figure 7. BER Comparison of Channel (a) for Different Estimators

By varying the SNR level, the performance of bit error rate (BER) comparison for different estimators is shown in Figure 6 and Figure 7 under two channel conditions to further test the performance of the proposed algorithm. It is shown that the proposed method leads to a better performance than LS method and OMP method. This is because that our method is deduced based on SpaRSA method, which can offer the optimal solution to the $\ell_{2}-\ell_{1}$ formulation of channel estimation. 
Table I reports the average CPU times required by the algorithms tested, where SNR is set to be $35 \mathrm{~dB}$. The experiments are performed under the aforementioned two channels using MATLAB on the computer with a CPU of Intel Core i5 and 2.0GB RAM. These results show that the proposed method has the fastest speed among these channel estimators. LS is slightly faster than OMP, and the proposed algorithm is clearly faster than LS and OMP. Since OMP cannot always guarantee successful sparse recovery and sometimes it runs out of all matrix columns, the time cost of OMP is the highest among these channel estimators. The proposed method pre-processes the initial channel estimates and then only a few of channel taps are re-estimated. This strategy reduces the computational operations of SpaRSA method. Hence, the proposed method costs less time than SpaRSA method.

Table 1. Table Label Average CPU Times of Channel Estimators

\begin{tabular}{ccc}
\hline \multirow{2}{*}{ Channel estimators } & \multicolumn{2}{c}{ CPU running times (in Seconds) } \\
\cline { 2 - 3 } & Channel (a) & Channel (b) \\
\hline LS & 23.615 & 23.437 \\
OMP & 33.248 & 34.125 \\
SpaRSA & 30.815 & 31.078 \\
Proposed method & 19.116 & 19.765 \\
\hline
\end{tabular}

\section{Conclusion}

In this paper, we propose a new algorithm based on SpaRSA algorithm for the estimation of sparse channels encountered by OFDM systems. This algorithm preserves the advantage of SpaRSA algorithm which is able to reach the global optimal solution to the $\ell_{2} \ell_{1}$ problem. It offers superior channel estimation performance by applying the threshold to determine the position of non-zero taps among the raw LS estimates which eliminates zero taps and helps the algorithm focus on the estimation of non-zero channel taps. Besides, introducing the noise effect into the regularization parameter overcomes the shortcoming of the original SpaRSA algorithm, which cannot reflect the change of channel noise. The influence of the regularization parameter is tested in the simulation. The simulation results also show that the proposed approach exhibits superior performance in terms of MSE, BER and average CPU running times when compared with the existing methods. Moreover, in contrast to the OMP method, our method does not require $a$ priori information about the number of non-zero channel taps.

\section{Acknowledgments}

This work is supported by the National Natural Science Foundation of China (11274253), the Scientific Research Program Funded by Shaanxi Provincial Education Department (14JK1673) and the Program of International Cooperation and Exchanges in Science and Technology Funded by Shaanxi Province (2016KW-033).

\section{References}

[1] S. Zhou and Z. Wang, "OFDM for Underwater Acoustic Communications", John Wiley \& Sons, ch.1, (2014).

[2] G. Gui, L. Xu and S.-Y. Matsushita, "Improved adaptive sparse channel estimation using mixed square/fourth error criterion”, Journal of the Franklin Institute, vol. 352, no. 10, (2015), pp. 4579-4594.

[3] C. Qi, Y. Huang, S. Jin and L. Wu, "Sparse channel estimation based on compressed sensing for massive MIMO systems", in Proc. IEEE International Conference on Communications, (2015), pp. 4558-4563.

[4] E. Habib, "Joint channel estimation and symbol detection for OFDM systems in rapidly time-varying sparse multipath channels", Wireless Personal Communications, vol. 82, no. 3, (2015), pp. 1161-1178. 
[5] G. Dziwoki and J. Izydorczyk, "Stopping criteria analysis of the OMP algorithm for sparse channels estimation", in Proc. 22nd International Conference on Computer Networks, vol. 522, (2015), pp. 250259.

[6] L. Yang, G. Ren and Z. Qiu, "Novel noise reduction algorithm for LS channel estimation in OFDM system with frequency selective channels", in Proc. IEEE Int. Conf. Comm. Syst., (2010), pp. 478-482.

[7] S. F. Cotter and B. D. Rao, "Sparse channel estimation via matching pursuit with application to equalization”, IEEE Trans. Commun., vol. 50, no. 3, (2002), pp. 374-377.

[8] N. L. Pedersen, C. N. Manchón and B. H. Fleury, "A fast iterative Bayesian inference algorithm for sparse channel estimation", in Proc. IEEE International Conference on Communications, (2013), pp. 4591-4596.

[9] B. Gao, Z. Xiao and C. Zhang, "Joint SNR and channel estimation for $60 \mathrm{GHz}$ systems using compressed sensing”, in Proc. IEEE Wireless Communication and Networking Conf., (2013), pp. 28962901.

[10] K. S. Priyanjali and A.V. Babu, "An improved least square channel estimation technique for OFDM systems in sparse underwater acoustic channel", in Proc. the 2014 International Conference on Advances in Computing, Communications and Informatics, ICACCI 2014, (2014), pp. 2521-2525.

[11] G. Z. Karabulut and A. Yongacoglu, "Sparse channel estimation using orthogonal matching pursuit algorithm", in Proc. IEEE Vehicular Technology Conference, (2004), pp. 3880-3884.

[12] C. Carbonelli, S. Vedantam and U. Mitra, "Sparse channel estimation with zero tap detection", IEEE Trans. Wireless Commun., vol. 6, no. 5, (2007), pp. 1743-1763.

[13] F. Wan, U. Mitra and A. Molisch, "The modified iterative detector/estimator algorithm for sparse channel estimation", in Proc. MTS/IEEE Oceans, (2010), pp. 1-6.

[14] Z. Jellali and L. N. Atallah, "Threshold-based channel estimation for MSE optimization in OFDM systems", in Proc. EURASIP, (2012), pp. 1618-1622.

[15] S. Daei, M. Babaie-Zadeh and C. Jutten, "A MAP-Based order estimation procedure for sparse channel estimation", in Proc. Latent Variable Analysis and Signal Separation - 12th International Conference, LVA/ICA 2015, (2015), pp. 344-351.

[16] S. J. Wright, R. D. Nowak and M. A. T. Figueiredo, "Sparse reconstruction by separable approximation", IEEE Transactions on Signal Processing, vol. 57, no. 7, (2009), pp. 2479-2493.

[17] S. Chen, D. Donoho and M. Saunders, "Atomic decomposition by basis pursuit", SIAM J. Sci. Comput., vol. 20, no. 1, (1998), pp. 33-61.

[18] J. Zhao, Y. Xu, C. Tian and F. Dong, "A fast sparse reconstruction algorithm for electrical tomography”, Measurement Science and Technology, vol. 25, (2014), pp. 1-14.

[19] T. Yaakov and D. L. Donoho, "Breakdown of equivalence between the minimal $\ell 1$-norm solution and the sparsest solution”, Signal Processing, vol. 86, no. 3, (2006), pp. 533-548.

[20] W. U. Bajwa, J. Haupt, A. M. Sayeed and R. Nowak, "Compressed Channel Sensing: A New Approach to Estimating Sparse Multipath Channels", in Proc. IEEE., vol. 98, (2010).

[21] B. Gao, Z. Xiao, C. Zhang, D. Jin and L. Zeng, "Sparse/dense channel estimation with non-zero tap detection for 60-GHz beam training", IET Communications, vol. 8, no. 11, (2014), pp. 2044-2053.

\section{Authors}

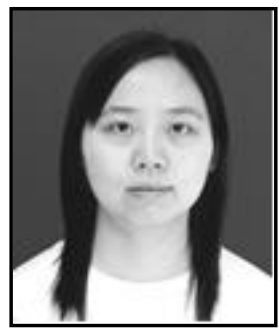

Xiaolin Shi, she received her M. Sc. and M. Phil. degrees from Northwestern Polytechnical University in 2005 and from City University of Hong Kong in 2006. She currently works as an associate professor of School of Electronics Engineering at Xi'an University of Posts \& Telecommunications, China. Her research interests include wireless communications and statistical signal processing.

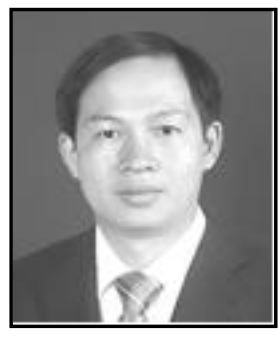

Yixin Yang, he received his Ph.D. degree from Northwestern Polytechnical University in 2002. He had been a Post-doctoral researcher from 2002 to 2004 at Nanyang Technological University, Singapore. Since June 2004, he has been working as a professor of School of Marine Science and Technology at Northwestern Polytechnical University, China. His research interests include underwater acoustics, acoustic signal processing, and acoustic system design. 
International Journal of Signal Processing, Image Processing and Pattern Recognition Vol. 10, No. 5 (2017) 